Research paper

\title{
Nutritional status of neurologically impaired children: Impact on comorbidity
}

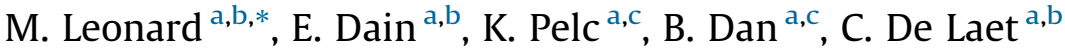 \\ a Université Libre de Bruxelles (ULB), 1050 Brussels, Belgium \\ ${ }^{\mathrm{b}}$ Service de maladies métaboliques et nutrition, Hôpital des Enfants Reine Fabiola, 1020 Brussels, Belgium \\ ${ }^{c}$ Inkendaal Revalidatieziekenhuis, 1602 Sint-Pieters-Leeuw, Belgium
}

\section{A R T I C L E IN F O}

\section{Article history:}

Received 25 June 2019

Received in revised form 24 September 2019

Accepted 11 November 2019

Available online 29 November 2019

\section{Keywords:}

Cerebral palsy

Malnutrition

Pressure ulcer

Pneumonia

Mid-upper arm circumference

Vitamin D deficiency

\begin{abstract}
A B S T R A C T
Background and aims: Malnutrition is common in neurologically impaired (NI) children. It is, however, illdefined and under-diagnosed. If not recognized and treated, it increases the burden of comorbidities and affects the quality of life of these children. The aim of this study was to characterize the nutritional status of a cohort of children followed up at a reference center for cerebral palsy (CP) in Brussels, Belgium, and to investigate possible links with the occurrence of comorbidities.

Material and methods: We conducted a single-center retrospective study including all the children followed up at the Inter-university Reference Center for Cerebral Palsy ULB-VUB-ULg. The data were obtained by reviewing medical files. Anthropometric measurements as well as the etiology of neurological impairment, comorbidities, feeding patterns, and laboratory test results were collected. The children were assigned a nutritional diagnosis according to the World Health Organization and Waterlow definitions.

Results: A total of 260 children with cerebral palsy were included, 148 males and 112 females. Their mean age was $10.9 \pm 4.3$ years. The gross motor function classification system (GMFCS) level was I for 79 children, II for 63 children, III for 35 children, IV for 33 children, and V for 50 children. Of the children, 54\% had a normal nutritional status, 34\% showed malnutrition, and $8 \%$ were obese; $38 \%$ had oropharyngeal dysphagia. The sensitivity of mean upper arm circumference of $<$ p10 to detect severe malnutrition was 95\%. Specific growth charts for $\mathrm{CP}$ were neither sensitive nor specific for predicting the risk of comorbidities. Malnutrition was associated with an increased risk of comorbidities (relative risk of $2.4[1.7 ; 3.4]$ ). It was also associated with the occurrence of pneumonia, pressure ulcers, and pathological bone fracture.

Discussion and conclusion: Children who are NI should be systematically and thoroughly screened for malnutrition, in the hope of offering early nutritional support and reduce comorbidities.
\end{abstract}

(c) 2019 French Society of Pediatrics. Published by Elsevier Masson SAS. All rights reserved.

\section{Introduction}

Neurological impairment comprise a heterogeneous group of disorders that primarily relate to the central nervous system, composed of the brain and spinal cord, affecting an individual's speech, motor skills, vision, memory, muscle actions, and learning abilities. Nutrition is a crucial component of health in neurologically impaired (NI) children. Nutritional evaluation and management of NI children are a constant challenge for health-care providers. However, malnutrition often remains under-diagnosed [1]. Malnutrition and obesity impact negatively the quality of life

\footnotetext{
* Corresponding author at: Service de maladies métaboliques et nutrition, Hôpital des Enfants Reine Fabiola, 1020 Brussels, Belgium

E-mail address: marie.leonard.be@gmail.com (M. Leonard).
}

and morbidity of these children. Malnutrition leads not only to stunting but also to muscle wasting and thus to a decreased ability to participate in everyday life or leisure activities. A decrease in axial muscle tone affects food intake and adds to the risk of choking and aspiration pneumonia. Moreover, the alteration of skin trophism along with sedentariness and macro- and micronutrient deficiencies promote poor healing and pressure ulcers [2-4].

Malnutrition in NI children is multifactorial. It is related in part to feeding difficulties associated with axial and face muscle weakness; it can be worsened by oropharyngeal dysphagia, or gastrointestinal disorders such as gastrointestinal reflux disease (GERD) and constipation, which are more prevalent in this population. Limited speech or intellectual disability can impair communication concerning hunger, thirst, or satiety, and thus lead to inadequate food or liquid intake. Moreover, various medications such as antiepileptic drugs can modify appetite and induce 
osteopenia through enzymatic induction of cytochrome CYP450, which enhances vitamin D metabolism $[2,5,6]$.

The prevalence of malnutrition in NI children is high; it is estimated to be $46-90 \%$ [2]. Caloric intake in this population is often low, which is justified in some cases by decreased energy expenditure due to lack of mobility. Yet low caloric intake can lead to malnutrition $[4,7]$.

Adapted strategies are required to evaluate the nutritional status of NI children. Measuring their weight and height using common measuring tools is often challenging. A weighting chair and a mobile measuring rod to measure the child in a supine position are often necessary. The patient may also be weighed while being carried in the arms of an adult. Height measurement may be skewed by musculoskeletal deformities, in which case it can be estimated from segmental measurements (tibial length, knee height) using appropriate equations [6]. However, this evaluation is somewhat imprecise, particularly for body mass index (BMI) calculation. Mean upper arm circumference (MUAC) and skinfold measurements allow for additional evaluation with data concerning body composition since they quantify the muscle and subcutaneous tissues $[8,9]$. Furthermore, laboratory analyses complement the evaluation assessing micronutrient status $[2,8]$.

In order to evaluate the approach of nutritional assessment in NI children and the comorbidities associated with malnutrition, we performed a retrospective study of the cohort of children followed up at the Inter-university Reference Center for Cerebral Palsy ULBVUB-ULg (Centre Interuniversitaire de Référence pour l'Infirmité motrice Cérébrale ULB-VUB-ULg [CIRICU]) in Brussels, Belgium. Our aim was to characterize the nutritional status in order to improve the follow-up scheme of these children.

\section{Material and methods}

\subsection{Participants}

We included all the NI children followed up at the CIRICU of the Queen Fabiola Children's University Hospital (HUDERF) in Brussels, Belgium on June 30, 2017.

\subsection{Data collection}

We reviewed the medical files of all patients actively enrolled in CIRICU on June 30, 2017 and collected data regarding age, sex, last anthropometric assessment (weight, height, growth curves, MUAC, tibial length, knee height), anamnestic data (etiology of neurological impairment, comorbidities, gross motor function classification system [GMFCS] level, food inquiry including type of diet and difficulties with meals), laboratory test data (complete blood count, iron status, serum protein level, serum albumin level, vitamin D level, and zinc level). We also noted the outcome of eventual orthopedic surgical interventions in terms of complications (wound infection, respiratory infections, urinary infections, and pressure ulcers).

\subsection{Data processing}

Data were anonymized.

If height measurement was unavailable for a child but segmental measurements were recorded, we estimated the height using the equations published by Scarpato et al. [6].

\subsubsection{Units}

Weight, height, and BMI were expressed as $z$-scores according to the World Health Organization (WHO) charts. The weight-forheight (WFH), i.e., the ratio between the current weight of a child and the expected weight for a defined height, was expressed as a percentage.

The weight, height, and BMI were also plotted on the specific charts for children with cerebral palsy (CP), according to their GMFCS level [10]. They were expressed in percentile ranges. Weight was classified as out of or in the red zone, which is below p5 for GMFCS I and II and below p20 for GMFCS III to V. This red zone was associated with an increased risk of comorbidities in the cohort from which the charts were built [11].

The MUACs were plotted on the Centers for Disease Control and Prevention $(C D C)$ charts [12], because although WHO data were collected for children up to 19 years [13], charts were not available for children over 5[14]. The values are expressed in percentile ranges, and were considered too low if they were $<\mathrm{p} 10$, as recommended by the guidelines of the European Society for Pediatric Gastroenterology Hepatology and Nutrition (ESPGHAN) [15].

\subsubsection{Definitions of nutritional status}

On the basis of their anthropometric data, a nutritional diagnosis was attributed to the children. Malnutrition was defined in our cohort using a combination of BMI $z$-score and Waterlow index [16].

Acute malnutrition was defined as a BMI $z$-score of $<-2$ standard deviation (SD), and/or a WFH of $<90 \%$. It was considered moderate if BMI was between -2 and -3 SD and/or WFH was between 80 and $89 \%$, and severe if BMI was $<-3$ SD and/or $\mathrm{WFH}<80 \%$. In the case of weight loss or stagnation during the previous 6 months, the subject was also considered acutely malnourished.

Chronic malnutrition was defined as a height $z$-score of $<-2$ SD for ambulant children (GMFCS I-III). However, we considered that it was not correct to apply this definition to wheelchair-bound children (GMFCS IV and V) as they have a lower growth [17]. We therefore used a lower threshold of $<-3$ SD for children in GMFCS levels IV and V. The malnutrition was defined as moderate between -2 and -3 SD for GMFCS I-III and between -3 and -4 SD for GMFCS IV and V, and as severe below -3 SD for GMFCS I-III and below -4 SD for GMFCS IV and V.

Obesity was defined using WFH for children under 5 years of age according to the WHO guidelines, with a threshold of $120 \%$, and using the BMI $z$-score for children over 5 years of age with a threshold of $>2$ SD.

If the anthropometric data were incomplete for a child but the available data were within the normal range, we considered that the child was neither malnourished nor obese, and had a normal nutritional status.

\subsection{Endpoints}

The primary endpoints were the description of the nutritional status and feeding pattern of this single-center cohort of NI children, and to establish a correlation between the nutritional status and the occurrence of comorbidities.

The secondary endpoints were to evaluate the utility of GMFCSspecific growth charts and of the MUAC measurement in nutritional evaluations. We also looked for a correlation between macro- and micronutrient deficiencies and the occurrence of comorbidities.

Finally, we evaluated the role of nutritional status in postorthopedic surgery outcome.

\subsection{Statistical analysis}

Statistical analysis was performed using the Statistica ${ }^{\circledR}$ software (version 13.2, Tulsa, Oklahoma). We used 
$2 \times 2$ contingency tables and performed $\mathrm{Chi}^{2}$ tests when the expected values were $>5$, or unilateral Fisher or $\mathrm{Chi}^{2}$ tests with Yates correction if one of the expected values was $<5$. We also used linear regressions and mean comparisons via unequal variances $t$ test. The normality of distribution was tested using a Shapiro-Wilk test. The significance level of $P$ was set at 0.05 for each of these analyses. Subgroup analyses were performed in order to address potential biases of positive findings as needed.

\section{Results}

\subsection{Data obtained}

A total of 260 children were included; 148 were males and 112 females, aged 18 months to 18 years. Their mean age was $10.9 \pm 4.3$ years. Weight was recorded for 251 children, height for 209. Height was estimated based on measurements of segments for an additional 20 patients. BMI was calculated for 218 children and WFH for 206. The last anthropometric data dated from the previous 12 months for 165 children (63\%), between 1 and 5 years for 72 children (28\%), between 5 and 10 years for 14 children (5\%), and more than 10 years in two children (1\%). No anthropometric data were found for seven subjects (3\%).

For 53 children (20\%), there was a specific nutrition follow-up in addition to CIRICU follow-up.

\subsection{Cohort characteristics}

The etiology of the neurological impairment is detailed in Table 1.

The severity of motor disability was categorized as GMFCS I for 79 children, GMFCS II for 63 children, GMFCS III for 35 children, GMFCS IV for 33 children, and GMFCS V for 50 children.

We confirmed that non-ambulant children (GMFCS IV and V) were significantly shorter with a mean height of $-2.3 \pm 1.8 \mathrm{SD}$ compared with walking children (GMFCS I-III), whose height was $-0.7 \pm 1.5 \mathrm{SD}$ (normal distribution, unequal variances $t$ test, $\left.P<10^{-4}\right)$.

\subsubsection{Prevalence of malnutrition}

In all, 55 children had acute malnutrition (28 moderate, 25 severe, two unclassified), 47 children had chronic malnutrition (29 moderate, 18 severe) (Fig. 1); 13 were malnourished both acutely and chronically (mixed malnutrition). A total of 22 children

Table 1

Etiology of neurological impairment $(n=260)$.

\begin{tabular}{ll}
\hline Etiology & Number of children \\
\hline Preterm birth, $n(\%)$ & $88(34)$ \\
Prenatal stroke, $n(\%)$ & $47(18)$ \\
Genetic or malformative disease, $n(\%)$ & $48(18)$ \\
Postnatal stroke, $n(\%)$ & $10(4)$ \\
Congenital heart disease & 6 \\
Sickle cell disease & 1 \\
Myocarditis & 1 \\
Cardiac arrest & 1 \\
Systemic lupus erythematosus & 1 \\
Perinatal asphyxia, $n$ (\%) & $30(12)$ \\
Other, $n$ (\%) & $37(14)$ \\
Shaken baby syndrome & 4 \\
Epileptic encephalopathy & 4 \\
Congenital infection & 5 \\
Kernicterus & 2 \\
Meningitis/encephalitis & 6 \\
Sequelae of brain cancer & 1 \\
Unknown & 15 \\
\hline
\end{tabular}

were obese; one of these also had chronic malnutrition. A normal nutritional status was found for 140 children. A nutritional diagnosis could not be attributed to 10 children: seven with no anthropometric data recorded; two with a very low height ( -4.1 SD in a child with Bohring-Opitz syndrome associated with epileptic encephalopathy but high BMI $z$-score and high WFH; and $-7.0 \mathrm{SD}$ for a child with holoprosencephaly but normal BMI $z$-score and normal WFH) that was considered part of their syndrome; one with a weight of $-3.4 \mathrm{SD}$ and no record of height.

The prevalence of malnutrition was significantly lower in the GMFCS I group compared with the GMFCS II-V groups and significantly higher in GMFCS V compared with GMFCS I-IV. Severe malnutrition was also significantly more prevalent in the GMFCS V group (Fig. 2).

\subsubsection{Prevalence of oropharyngeal dysphagia}

Oropharyngeal dysphagia, defined as difficulty with chewing or swallowing, was present in 98 patients (38\%). In the GMFCS I group, $76 \%$ reported having a normal diet, while $22 \%$ reported having some difficulties with swallowing or prolonged meal duration. Only two of them (3\%) needed modification of the diet texture (mixed, soft, finely cut). The prevalence of oropharyngeal dysphagia increased according to the GMFCS level. Only children with GMFCS IV-V levels required enteral nutrition: $6 \%$ and $40 \%$ of them, respectively (Fig. 3).

Choking episodes were reported by parents for $28 \%$ of the children. They were more prevalent in the GMFCS V group (60\%), and were more often described as "frequent episodes" than in the other GMFCS levels (Fig. 4). It should be noted that among the 24 children with frequent episodes of choking, five still reported having a normal diet without modification of texture.

In total, $73 \%$ of the children with obesity were free from symptoms of oropharyngeal dysphagia, $18 \%$ had some difficulty swallowing or prolonged meal duration, and 9\% required a modified-texture diet; $13 \%$ also reported occasional choking. These proportions are not different from the rest of the cohort, taking into account the degree of motor disability.

\subsection{Comorbidities}

There was a documented history of pneumonia in 52 children (20\%), among whom nine (3.5\%) required (invasive or noninvasive) respiratory support in a pediatric intensive care unit. Furthermore, 39 children (15\%) had GERD and 40 (15\%) showed constipation. Three patients (1.2\%) presented with pressure ulcers, and four (1.5\%) with pathological bone fractures.

\subsubsection{Factors influencing the overall rate of comorbidities}

The overall rate of comorbidities increased according to GMFCS level (Fig. 5).

Malnutrition (acute and chronic) was also associated with an increased risk of comorbidities with and relative risk (RR) of 2.4 [95\% confidence interval (95\% CI): 1.7; 3.4] (Fig. 6). It was even more increased in cases of severe malnutrition ( $\mathrm{RR}=3$ [95 CI: 2.1; 4.3]). The analysis of the GMFCS subgroups yielded similar results for the GMFCS I and III levels (Fisher test, $P<0.05$ ). The analysis of subgroups according to the type of malnutrition (acute or chronic) yielded similar results $\left(\chi^{2}\right.$ test, $P<10^{-4}$ ). We did not observe a significant difference in the occurrence of comorbidities between children with obesity and those with normal nutritional status.

\subsubsection{Factors influencing the rate of pneumonia}

The occurrence of pneumonia was strongly correlated with frequent choking episodes, independently of GMFCS level, with an RR of 5.1 [95\% CI: 3.1; 8.3] (Fig. 7). Occasional choking did not significantly increase the risk of pneumonia. 


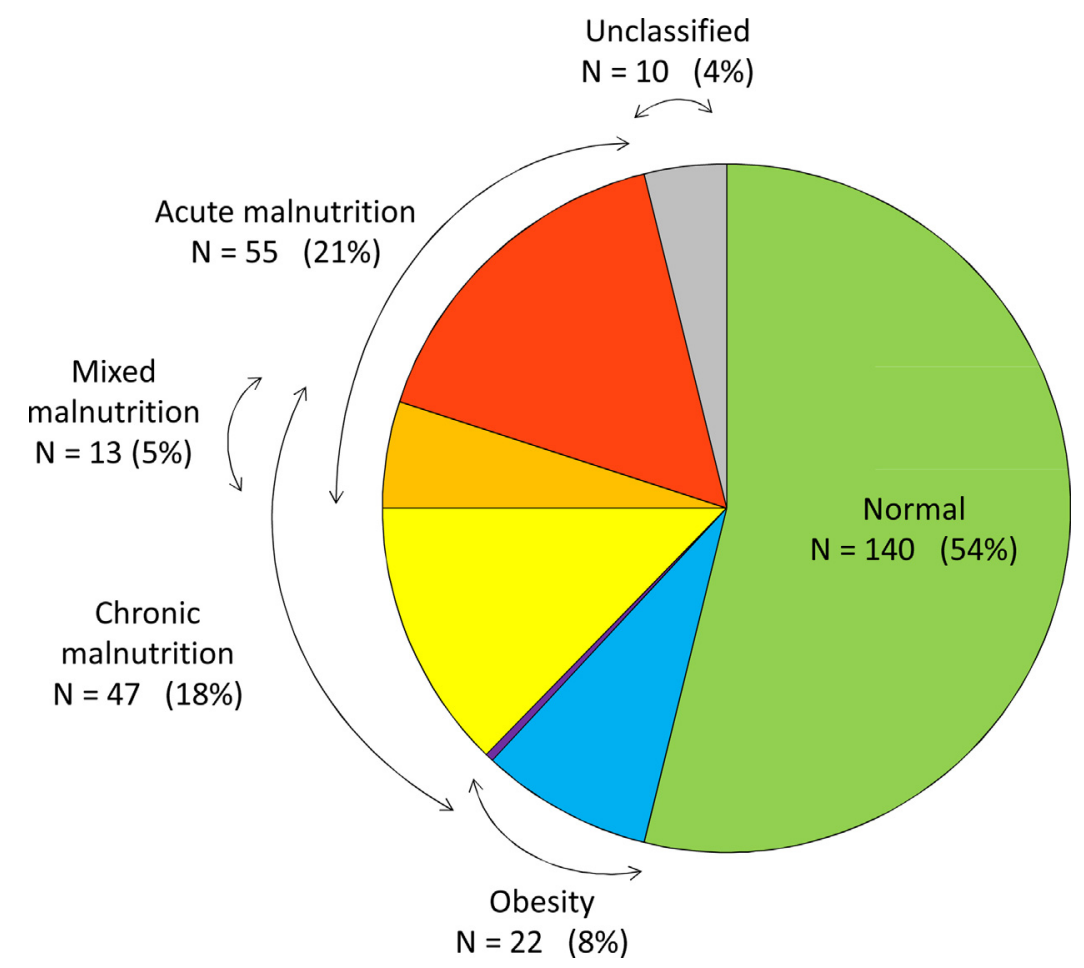

Fig. 1. Distribution of nutritional status $(n=260)$.

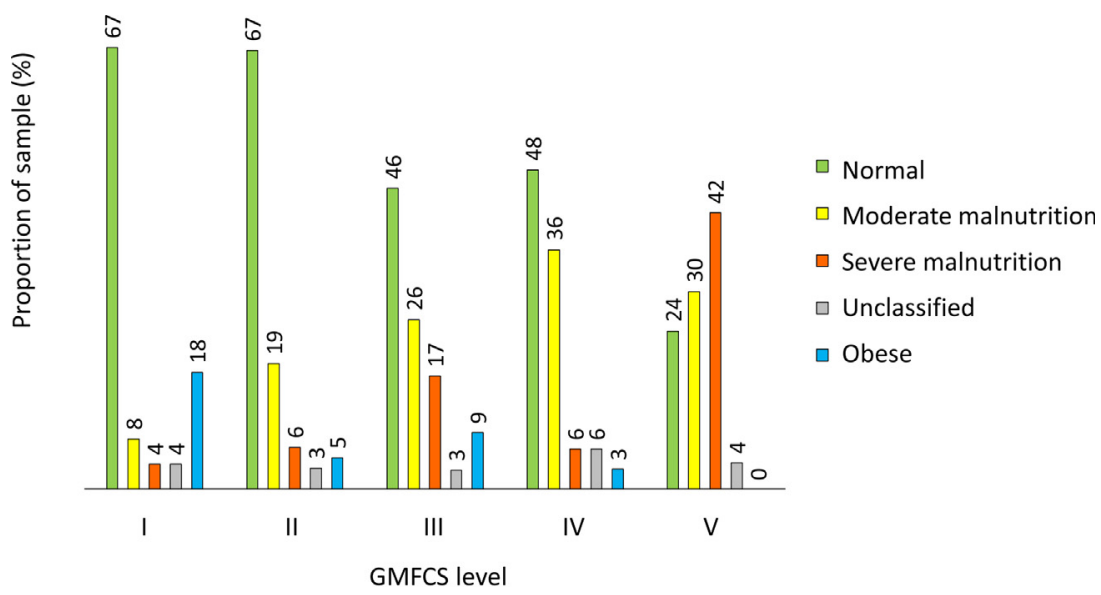

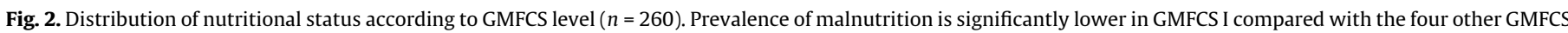

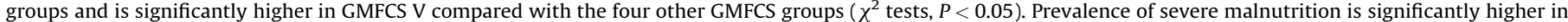
GMFCS V compared with GMFCS I-IV groups ( $\chi^{2}$ tests, $\left.P<0.05\right)$. GMFCS: Gross motor function classification system.

Malnutrition was also a risk factor for pneumonia (RR $=3[95 \%$ CI: $1.6 ; 5.4$ ] for moderate malnutrition; $\mathrm{RR}=3.8$ [95\% CI: $2.1 ; 7.0$ ] for severe malnutrition with $\chi^{2}$ tests, $P<0.05$ ), even after neutralizing the confounding factor "choking" via a subgroup analysis (Fig. 7). In this subgroup analysis, malnutrition was a risk factor for pneumonia in children having no choking episodes or occasional choking, but not those with frequent choking episodes.

In total, 14 patients with missing data regarding choking were excluded from these analyses. They had significantly more episodes of pneumonia than the rest of the cohort $\left(\chi^{2}\right.$ test, $P=0.003$ ).

\subsubsection{Factors influencing the rate of pressure ulcers}

The occurrence of pressure ulcers was significantly correlated with malnutrition in the full cohort, and in subgroups according to the type of malnutrition (significant difference in severe malnutrition, acute malnutrition, and mixed malnutrition) with Fisher tests, $P<0.05$.

\subsubsection{Factors influencing the rate of pathologic bone fractures}

Pathological bone fractures occurred significantly more often in the case of mixed malnutrition (Fisher test, $P=0.015$ ).

\subsection{Comorbidities occurring after orthopedic surgery}

Of the children, 74 had had orthopedic surgery; 11 of them underwent nutrition follow-up and four had enteral nutritional support. Eight patients (10.8\%) developed postoperative complications: pressure ulcers $(n=2)$, pneumonia $(n=4)$, urinary tract infection $(n=1)$, and complex regional pain syndrome $(n=1)$. The rate of complications was significantly higher after a bone surgery 


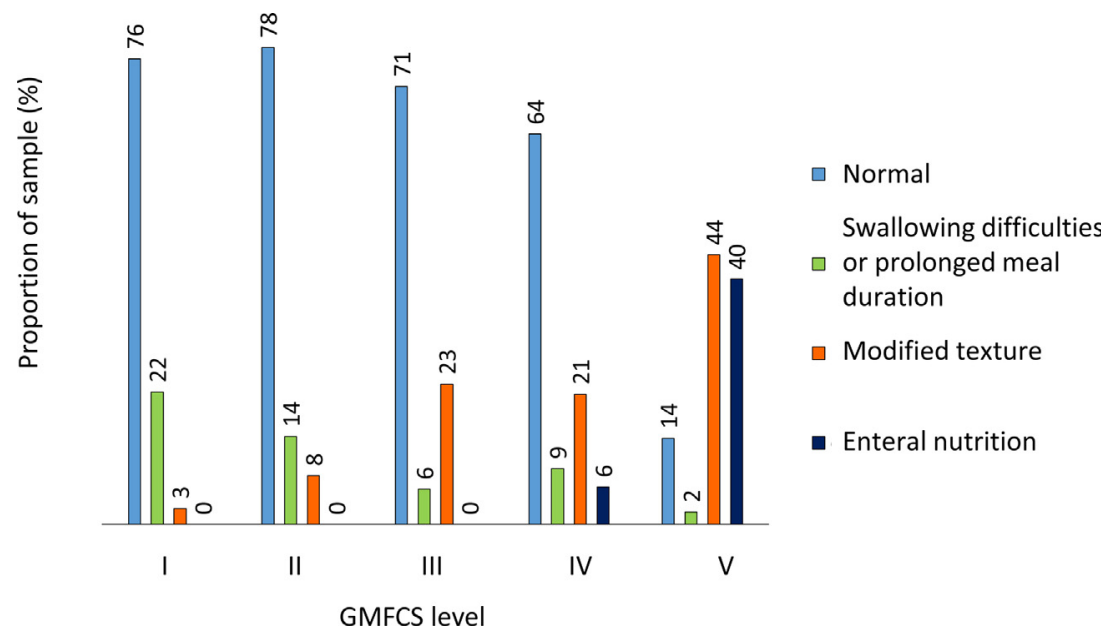

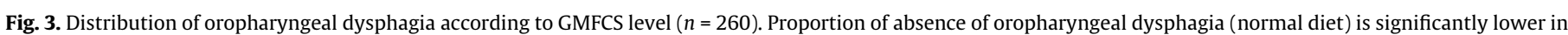

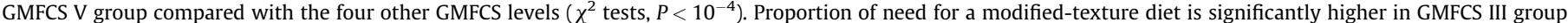
compared with GMFCS I (Fisher test, $P=0.001$ ) and GMFCS II groups (Fisher test, $P=0.040$ ). GMFCS: Gross motor function classification system.

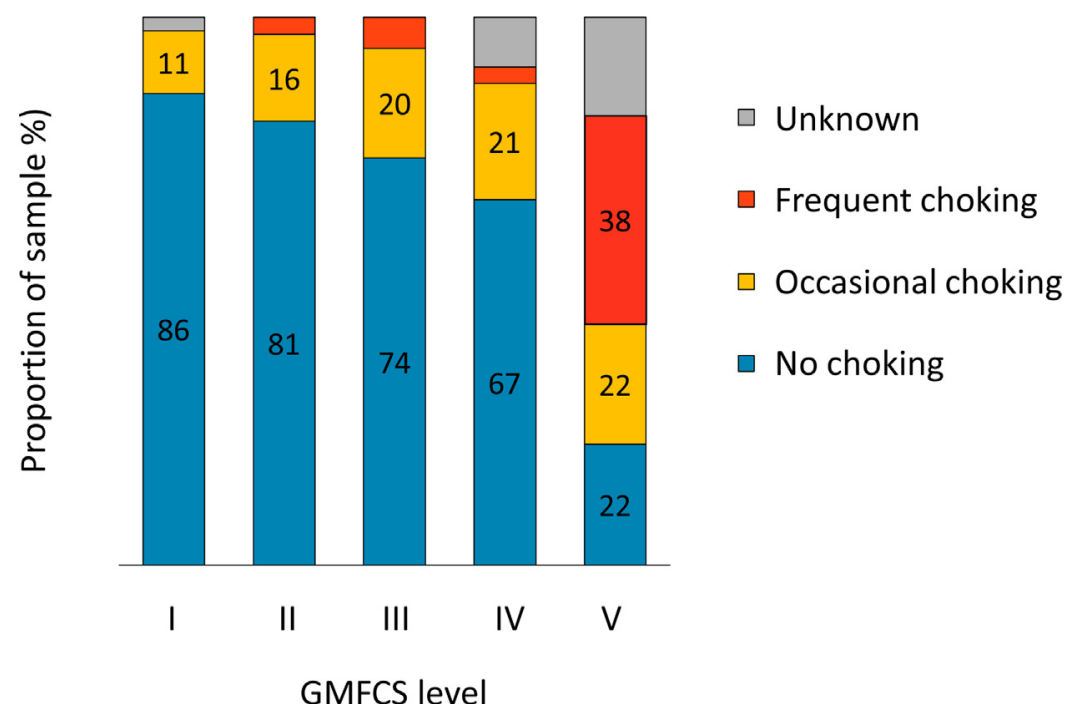

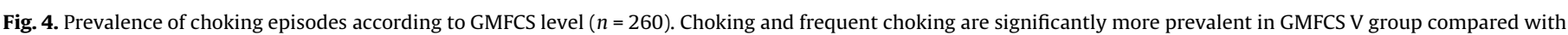
GMFCS I-IV groups $\left(\chi^{2}\right.$ tests, $\left.P<10^{-4}\right)$. GMFCS: Gross motor function classification system.

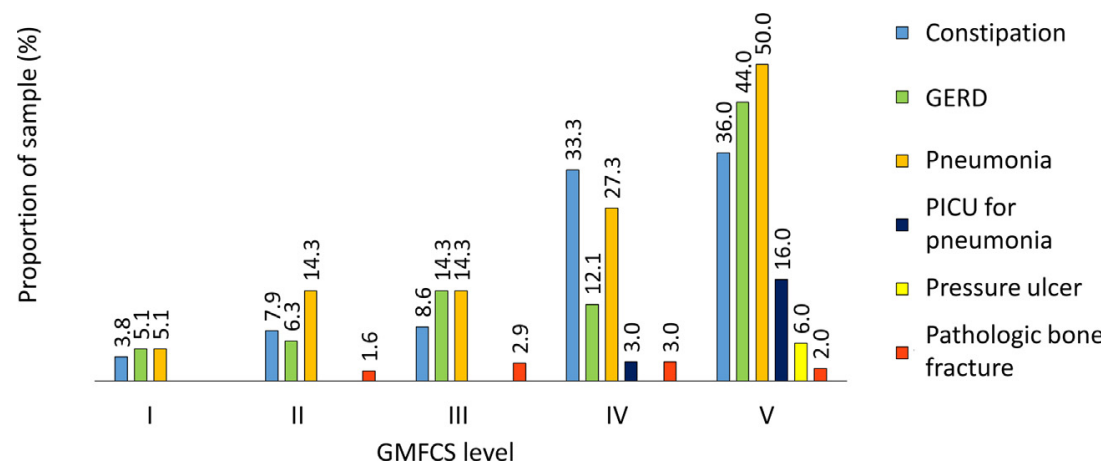

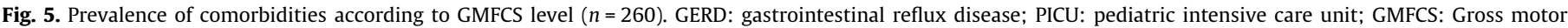
function classification system. Rate of comorbidities is significantly higher in GMFCS IV and V groups compared with GMFCS I-III groups $\left(\chi^{2}\right.$ tests, $\left.P<0.5\right)$.

(arthrodesis or osteotomy) than after a soft tissue surgery (tendon transposition or lengthening): $25 \%$ vs. $2.9 \%$ (Fisher test, $P=0.027$ ). There was, however, no significant difference in the rate of complications according to nutritional status or nutrition follow-up.

\subsection{Utilization of the red zone of the specific charts for $C P$}

The data of 246 children were plotted on the GMFCSspecific growth charts. Among them, 21 were in the red zone for weight. 


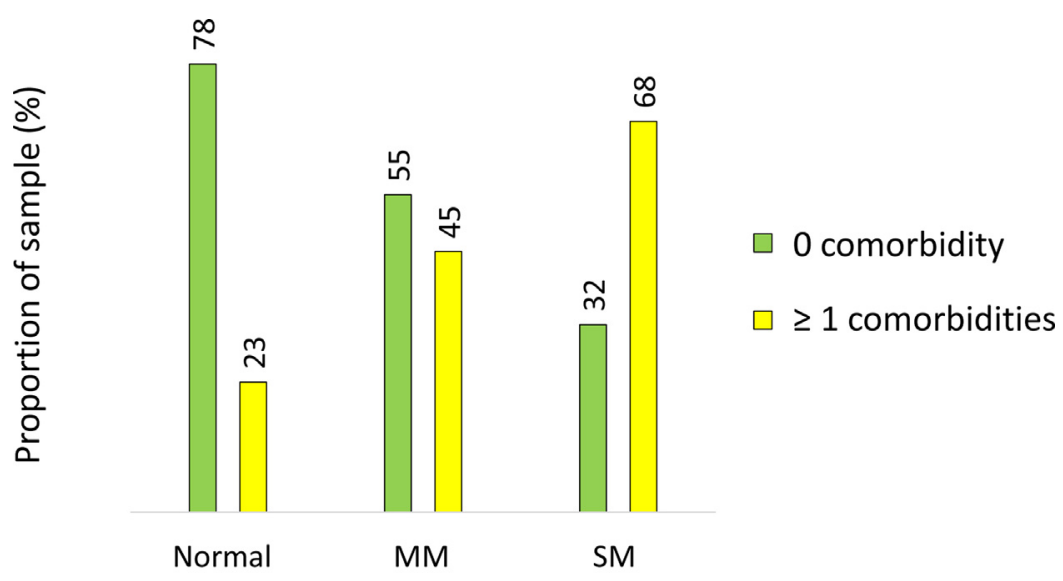

\section{Nutritional status}

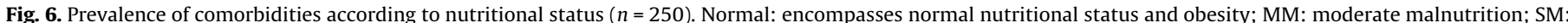

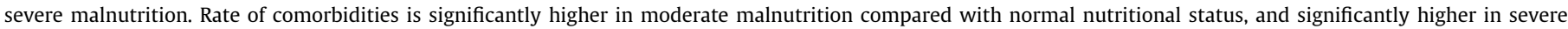
malnutrition compared with moderate malnutrition $\left(\chi^{2}\right.$ tests, $\left.P<0.05\right)$. NB: children with unknown nutritional status were excluded from this analysis.
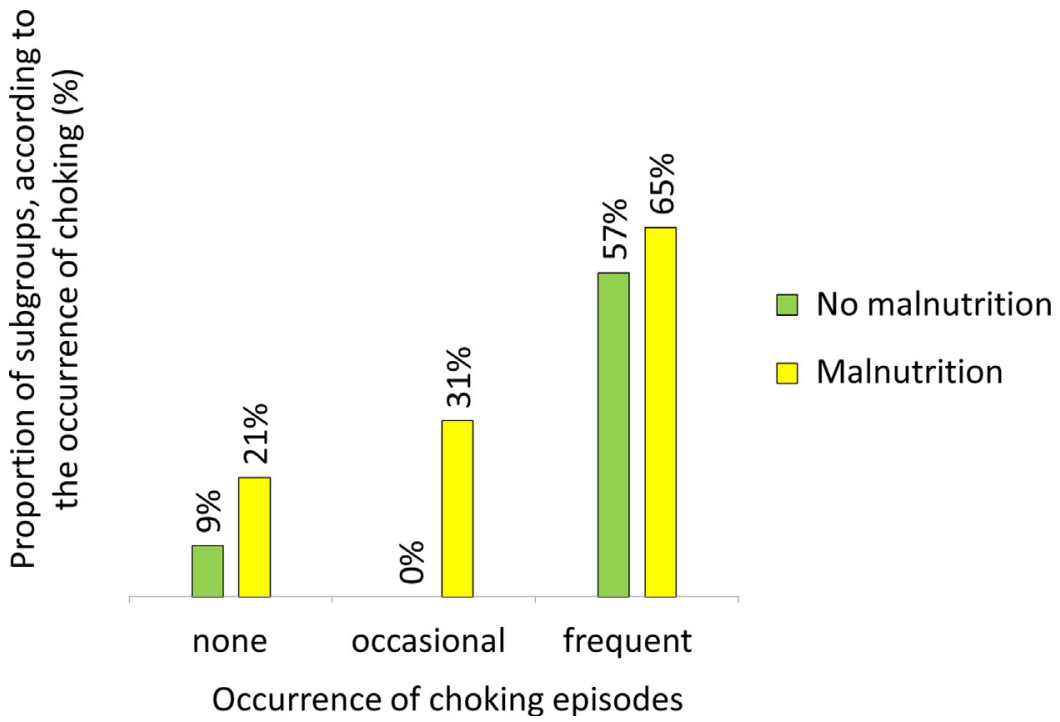

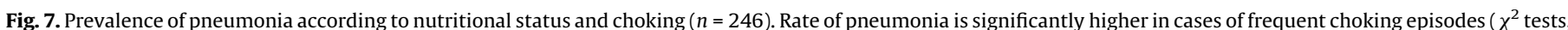

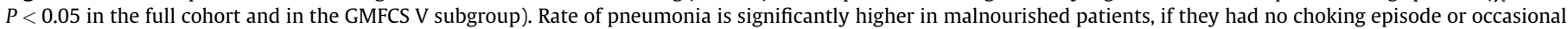

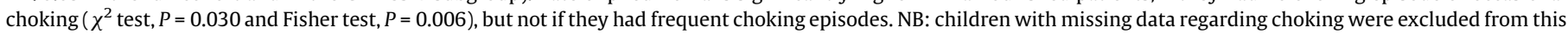
analysis; GMFCS: Gross motor function classification system.

In comparison with our definitions of malnutrition using WHO and Waterlow criteria, 17 of these 21 patients in the red zone were considered severely malnourished, three had moderate malnutrition, and one had a normal nutritional status (the aforementioned child with syndromic short stature). However, 68 malnourished children, including 21 who were severely malnourished, were above this red zone of the GMFCS growth charts.

Of the 21 children in the red zone, 16 (76\%) had comorbidities, including the three children with pressure ulcers. Of the 68 malnourished children not classified in the red zone, 33 (48\%) also had comorbidities, including four children admitted to a pediatric intensive care unit because of pneumonia and one child with pathological bone fracture.

\subsection{Utilization of the MUAC}

In total, 35 of 82 patients whose MUAC was measured had an MUAC of $<$ p10. Having an MUAC of $<$ p10 was significantly associated with malnutrition, including isolated acute and isolated chronic malnutrition $\left(n=82, \chi^{2}\right.$ tests, $\left.P<0.05\right)$. The sensitivity of an MUAC of $<$ p10 was particularly good in detecting severe malnutrition. For overall severe malnutrition, the sensitivity was $95 \%$ and specificity $75 \%$; it was $100 \%$ and $72 \%$ concerning severe acute malnutrition, respectively; and $90 \%$ and $64 \%$ for severe chronic malnutrition, respectively.

\subsection{Macro- and micronutrient deficiencies}

Iron deficiency was found in 43 of the 114 children who were tested (38\%). Anemia was found in 24 of 171 patients for whom a total blood count was performed (14\%), 83\% of whom had iron-deficiency anemia. We could not find a significant correlation in this cohort between iron status and type of diet, nutritional status or pressure ulcers. Anemia was not correlated with pressure ulcers either.

Total serum protein and serum albumin were identified in 129 children. Nine of them had hypoproteinemia (low protein 


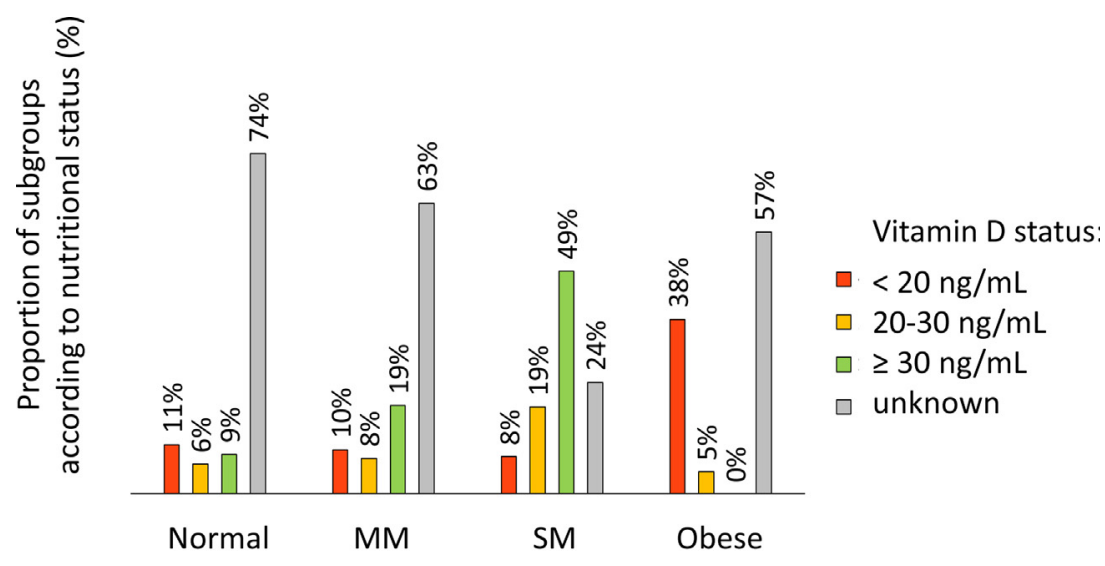

Nutritional status

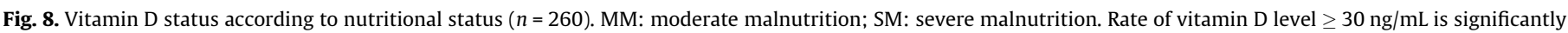

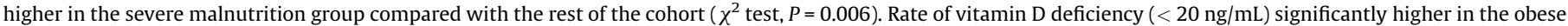

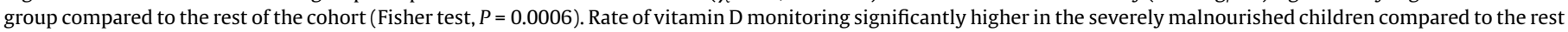
of the cohort $\left(\mathrm{Chi}^{2}\right.$ test, $\left.\mathrm{P}<10^{-4}\right)$.

and/or low albumin). Malnourished children had a higher risk of hypoproteinemia (RR 9.1 [95\% CI: 1.2; 70.5], Fisher test, $P=0.010$ ). Hypoalbuminemia was a major risk factor for the occurrence of pressure ulcers with an RR of 41 [95\% CI: 4.3; 391.5] (Fisher test, $P=0.005$ ).

Vitamin D was monitored in 94 patients. The level was $<20 \mathrm{ng} / \mathrm{mL}(<50 \mathrm{mmol} / \mathrm{L})$ in 32 children (34\%), between 20 and $30 \mathrm{ng} / \mathrm{mL}(50-75 \mathrm{mmol} / \mathrm{L})$ in 21 children $(22 \%)$ and $\geq 30 \mathrm{ng} / \mathrm{dL}$ ( $\geq 75 \mathrm{mmol} / \mathrm{L}$ ) in 41 children (44\%). Vitamin D level was well correlated (but inversely) with the nutritional status (Fig. 8). In $64 \%$ of the severely malnourished patients had an optimal vitamin $\mathrm{D}$ level $(\geq 30 \mathrm{ng} / \mathrm{mL}$ ), while none of the obese children had an optimal level. On the contrary, $89 \%$ of the obese children had vitamin D deficiency ( $<20 \mathrm{ng} / \mathrm{mL}$ ), which is an RR of 3.1 [95\% CI: 2.1 ; 4.7] to be vitamin D-deficient if obese in our cohort. Vitamin D was monitored in $76 \%$ of the severely malnourished children compared with $30 \%$ for the rest of the cohort, which is a statistical difference. Vitamin D deficiency was not associated with the occurrence of pathological bone fracture. Out of the four patients who had a pathological bone fracture, two had a vitamin D level of $>30 \mathrm{ng} / \mathrm{mL}$, one of $<20 \mathrm{ng} / \mathrm{mL}$, and one was unknown. Three of these children were on antiepileptic drugs.

Zinc levels were monitored in 40 children and $52 \%$ of them had a zinc deficiency. No correlation was found with the nutritional status. No increase in comorbidities, in particular pressure ulcers, was noted in the children with zinc deficiency in this cohort.

\section{Discussion}

\subsection{How to define malnutrition in NI children}

Malnutrition is difficult to define in all groups of people, since it is a dynamic condition, implying intake that is inadequate to allow for homeostasis and physical activity, and in children, growth [18]. Anthropometric data provide the most practical way to approach the concept of malnutrition in clinical practice.

Given the specific phenotype of NI children, there is no clear definition of malnutrition in this population, as highlighted in the guidelines from ESPGHAN [15]. ESPGHAN and its North American equivalent NASPGHAN suggest using BMI to track acute malnutrition, although imprecision in height measurements makes this index somewhat inappropriate in some children; the alternative index is the WFH $[15,19]$.

It was difficult to establish cut-offs in our cohort, resulting in a composite definition of acute malnutrition using the WHO and Waterlow definitions (with BMI $z$-score and WFH percentages) and a dynamic component that was the absence of weight gain during the previous 6 months. We arbitrarily decided to use a different threshold of height $z$-score to define chronic malnutrition in non-ambulant children (GMFCS IV and V) since their growth potential is intrinsically altered by their pathology. We were reassured with this choice because they were overall significantly shorter than the rest of the children, with a mean height under $-2 \mathrm{SD}$. This is also supported by the specific growth charts for children with $\mathrm{CP}$, in which median height drops according to the GMFCS level [11].

Because of the challenges in measuring anthropometric parameters in NI children, the nutritional evaluation must be multimodal. Commonly used anthropometric data, such as BMI, $\mathrm{WFH}$, and height-for-age, are the basis of the evaluation.

According to our results, the red zone for weight depicted in the specific charts for children with $\mathrm{CP}$ is a criterion of the severity of malnutrition, but is not sufficient to predict the risk of comorbidities.

The MUAC measurement provides additional information on body composition. It quantifies bone, muscle, and the adipose panicle (representative of the energetic balance). Its interpretation with standards calculated on a reference population is biased in NI children, whose body composition is altered owing to their pathology (muscle wasting and osteopenia, partly as a consequence of lack of mobility). The MUAC was measured in a third of our cohort, and analyzed with CDC standards making our results subject to caution. However, it seems to be a good screening tool for malnutrition, with a very high sensitivity regarding severe malnutrition, and it is easily measured with a simple tape, even on children with reduced mobility. WHO charts for MUAC are now available for children until the age of 19 and should also be evaluated in this setting [13].

Skinfold measurements were not recorded in our study because of the scarcity of available data. They are, however, regarded as a better measure of the adipose panicle and provide the best estimation of body composition [20]. They require access to an adipometer, and appropriate training for accurate measurements. 


\subsection{Prevalence of malnutrition}

The prevalence of malnutrition in our cohort is comparable to that described in previous reports, both in terms of undernutrition and obesity $[2,21]$. Malnutrition is highly prevalent, with more than a third of children featuring some kind of malnutrition, enhancing the need for systematic nutritional screening in $\mathrm{NI}$ children.

\subsection{Prevalence and consequences of oropharyngeal dysphagia}

The rate of dysphagia in NI children has been reported to be $85 \%$, if objectively evaluated during a meal with a score attributed by a speech therapist trained in swallowing/chewing evaluation [22]. If evaluated based on parents' report alone, as is the case in our study, the rate of dysphagia drops to about $26 \%$, according to Lopes et al. [23] or by 50\% compared with a speech therapist observation, according to Benfer et al. [24].

The texture of the food consumed by the children in our cohort is also similar to that described in the cohort of Benfer et al., in which $81 \%$ of the GMFCS I and II children had a normal diet, versus $22 \%$ for the GMFCS IV and V [24].

Given the strong correlation between swallowing difficulties, especially choking, and the occurrence of pneumonia, it seems important to systematically investigate the quality of swallowing in NI children through their history. Those who report difficulties with food or pneumonia should also be objectively evaluated in order to implement strategies that could make the meal times safer (modified textures of food, good installation for eating, start of enteral feeding).

\subsection{Link between malnutrition and comorbidities}

We cannot draw conclusions on the rate of comorbidities in our cohort, since the study design is based on a file review. However, malnutrition in NI children appears to be per se a risk factor of comorbidities, in particular pneumonia and pressure ulcers, even when taking into account confounding factors such as severity of handicap or choking. It is a strong argument in support of systematic screening for malnutrition in all NI children.

Comorbidities of the gastrointestinal tract (GERD and constipation) are highly prevalent. Previous studies report a rate twice or three times superior to ours, for the reasons explained above [2]. Even if these comorbidities do not seem to be influenced by nutritional status, they can impede nutritional care and cause significant discomfort. They should therefore be sought for and treated.

We could not establish a relation between nutritional status and postsurgical comorbidities. This is probably due to a relatively small cohort of children having had orthopedic surgery and to putative missing data. Such complications of surgery have previously been reported in several observational studies in adults $[25,26]$. The scheduling of orthopedic surgery in a NI child, especially if it is for a major surgery, should be combined with a nutritional evaluation if not done previously, and the nutritional status of the child should be restored if necessary prior to surgery. More prospective studies are needed in children to confirm the benefits of such therapeutic options.

\subsection{Macro- and micronutrient deficiencies}

Macro- and micronutrient deficiencies are abundant in our cohort when searched for, as previously reported [2], even though prevalence cannot be calculated owing to numerous missing data. These deficiencies occur in the setting of feeding difficulties and reduced energy needs in some children, with reduced volume of feeding and sometimes unbalanced macro- and micronutrient contents. They are not harmless. Iron deficiency is of course a risk factor for anemia.

We found an increased rate of hypoproteinemia in malnourished children and a large increase in the risk of pressure ulcers in those presenting with hypoalbuminemia (which actually reflects severe malnutrition).

We did not observe a significant relationship between vitamin $\mathrm{D}$ status and pathological bone fracture, but we did not evaluate the calcium intake of our subjects. Interestingly, vitamin D deficiency was monitored much more often in malnourished children, but was significantly more prevalent in obese children. One explanation is that malnourished children are more spontaneously given supplementation with vitamin D. As for obese children, several studies have shown a correlation between obesity and vitamin D deficiency in children without disabilities [27,28]. The pathophysiology behind this link has not been very well explained so far.

Thus, when evaluating NI children for malnutrition, macro- and micronutrients have to be monitored, especially in patients with feeding difficulties and in malnourished children. Vitamin D should probably be systematically supplemented in most NI children, regardless of their nutritional status, since they are a group at risk for vitamin D deficiency owing to their lack of mobility and sun exposure [29]. Appropriate calcium intake should be achieved by dairy consumption or supplementation.

\section{Conclusion}

NI children are at high risk of malnutrition. This leads to comorbidities and an altered quality of life, which can be prevented by efficient screening for the risk factors of malnutrition, for malnutrition itself, and by targeted and multidisciplinary care. More studies are needed to define biometric thresholds tailored to NI children.

In the setting of a multidisciplinary follow-up of NI children, a nutritionist physician should be involved from the start, before malnutrition occurs and becomes a problem for the child. NI children should be regularly measured and weighed, despite the practical difficulties, in order to screen the development toward malnutrition or obesity. MUAC should also be monitored as an additional screening tool, since it is easy and quick to measure, or skinfold thickness if adequate tools are available. If needed, the evaluation of swallowing should be performed by a trained speech therapist. A dietary evaluation should be obtained, in the form of a standardized questionnaire, in order to avoid unbalanced macroand micronutrient intake, which occurs much more frequently if the child has difficulties with meal times.

When a major surgery such as orthopedic surgery has to be scheduled, the child should be reassessed as part of a nutrition consultation in order to provide nutritional support when needed, and hopefully reduce the rate of postoperative complications.

\section{Ethics}

Approval from the Ethics Committee of HUDERF was obtained for this study. Since it was a retrospective survey, no informed consent was required according to Belgian legislation.

\section{Disclosure of interest}

The authors declare that they have no competing interest.

\section{Acknowledgments}

We want to thank Caroline De Longueville for her precious computer support and other advice, and the CIRICU team for their collaboration. 


\section{References}

[1] Dan B. Nutrition, brain function, and plasticity in cerebral palsy. Dev Med Child Neurol 2016;58:890.

[2] Penagini F, Mameli C, Fabiano V, et al. Dietary intakes and nutritional issues in neurologically impaired children. Nutrients 2015;7:9400-15.

[3] Samson-Fang L, Stevenson R. Identification of malnutrition in children with cerebral palsy: poor performance of weight-for-height centiles. Dev Med Child Neurol 2000;42:162-8.

[4] Kilpinen-Loisa P, Pihko H, Vesander U, et al. Insufficient energy and nutrient intake in children with motor disability. Acta Paediatr 2009:98:1329-33.

[5] Quitadamo P, Thapar N, Staiano A, et al. Gastrointestinal and nutritional problems in neurologically impaired children. Eur J Paediatr Neurol 2016;20:810-5.

[6] Scarpato E, Staiano A, Molteni M, et al. Nutritional assessment and intervention in children with cerebral palsy: a practical approach. Int J Food Sci Nutr 2017;68:763-70.

[7] Azcue M, Zello G, Levy L, et al. Energy expenditure and body composition in children with spastic quadriplegic cerebral palsy. J Pediatr 1996;129:870-6.

[8] Samson-Fang L, Bell K. Assessment of growth and nutrition in children with cerebral palsy. Eur J Clin Nutr 2013;67:S5-8.

[9] Kuperminc M, Gurka M, Bennis J, et al. Anthropometric measures: poor predictors of body fat in children with moderate to severe cerebral palsy. Dev Med Child Neurol 2010;52:824-30.

[10] Growth charts for children with cerebral palsy. Life expectancy; 2011. Available from: http://www.lifeexpectancy.org/articles/NewGrowthCharts.shtm [Accessed 9th August 2017].

[11] Brooks J, Day S, Shavelle R, et al. Low weight, morbidity, and mortality in children with cerebral palsy: new clinical growth charts. Pediatrics 2011;128:e299-307.

[12] Fryar C, Gu Q, Ogden C. Anthropometric reference data for children and adults: United States, 2007-2010. Vital Health Stat 11 2012;252:1-48.

[13] Mramba L, Ngari M, Mwangome M, et al. A growth reference for mid upper arm circumference for age among school age children and adolescents, and validation for mortality: growth curve construction and longitudinal cohort study. BMJ 2017;358:j3423.

[14] The WHO Child Growth Standards. World Health Organization; 2006/2007. Available from: http://www.who.int/childgrowth/standards/en/ for children up to 5 years, and https://www.who.int/growthref/en/ for children 5-19 years [Accessed 9th August 2017].
[15] Romano C, van Wynckel M, Hulst J, et al. European Society for Paediatric Gastroenterology, Hepatology and Nutrition guidelines for the evaluation and treatment of gastrointestinal and nutritional complications in children with neurological impairment. J Pediatr Gastroenterol Nutr 2017;65:242-64.

[16] Mehta N, Corkins M, Lyman B, et al. Defining pediatric malnutrition: paradigm shift toward etiology-related definitions. J Parenter Enteral Nutr 2013;37:460-81

[17] Grossberg R, Wolff J. Defining optimal growth in cerebral palsy: comparing apples to apples? Dev Med Child Neurol 2016;58:892-3.

[18] Cederholm T, Barazzoni R, Austin P, et al. ESPEN guidelines on definitions and terminology of clinical nutrition. Clin Nutr 2017;36:49-64.

[19] Marchand V, Motil K. NASPGHAN committee on nutrition. Nutrition support for neurologically impaired children: a clinical report of the North American Society for Pediatric Gastroenterology, Hepatology, and Nutrition. J Pediatr Gastroenterol Nutr 2006;43:123-35.

[20] Gurka M, Kuperminc M, Busby M, et al. Assessment and correction of skinfold thickness equations in estimating body fat in children with cerebral palsy. Dev Med Child Neurol 2010;52:e35-41.

[21] Pascoe J, Thomason P, Graham H, et al. Body mass index in ambulatory children with cerebral palsy: a cohort study. J Paediatr Child Health 2016;52:417-21.

[22] Benfer K, Weir K, Bell K, et al. Oropharyngeal dysphagia and gross motor skills in children with cerebral palsy. Pediatrics 2013;131:e1553-62.

[23] Lopes P, Amancio O, Araújo R, et al. Food pattern and nutritional status of children with cerebral palsy. Rev Paul Pediatr 2013;31:344-9.

[24] Benfer K, Weir K, Bell K, et al. Food and fluid texture consumption in a population-based cohort of preschool children with cerebral palsy: relationship to dietary intake. Dev Med Child Neurol 2015;57:1056-63.

[25] Legg J, Davies E, Raich A, et al. Surgical correction of scoliosis in children with spastic quadriplegia: benefits, adverse effects, and patient selection. EvidBased Spine-Care J 2014;5:38-51.

[26] Jevsevar D, Karlin L. The relationship between preoperative nutritional status and complications after an operation for scoliosis in patients who have cerebral palsy. J Bone Joint Surg Am 1993;75:880-4.

[27] Peterson C, Belenchia A. Vitamin D deficiency \& childhood obesity: a tale of two epidemics. Mo Med 2014;111:49-53.

[28] Smotkin-Tangorra M, Purushothaman R, Gupta A, et al. Prevalence of vitamin D insufficiency in obese children and adolescents. J Pediatr Endocrinol Metab 2007;20:817-23.

[29] Braegger C, Campoy C, Colomb V, et al. Vitamin D in the healthy European paediatric population. J Pediatr Gastroenterol Nutr 2013;56:692-701. 